\title{
Trampoline related injuries in children: risk factors and radiographic findings
}

\author{
Peter Klimek, David Juen, Enno Stranzinger, Rainer Wolf, Theddy Slongo \\ Bern, Switzerland
}

Background: Backyard trampolines are immensely popular among children, but are associated with an increase of trampoline-related injuries. The aim of this study was to evaluate radiographs of children with trampoline related injuries and to determine the risk factors.

Methods: Between 2003 and 2009, 286 children under the age of 16 with backyard trampoline injuries were included in the study. The number of injuries increased from 13 patients in 2003 to 86 in 2009 . The median age of the 286 patients was 7 years (range: 1-15 years). Totally $140(49 \%)$ patients were males, and $146(51 \%)$ females. Medical records and all available diagnostic imaging were reviewed. A questionnaire was sent to the parents to evaluate the circumstances of each injury, the type of trampoline, the protection equipment and the experience of the children using the trampoline. The study was approved by the Institutional Ethics Committee of the University Hospital of Bern.

Results: The questionnaires and radiographs of the 104 patients were available for evaluation. A fracture was sustained in 51 of the 104 patients. More than $\mathbf{7 5 \%}$ of all patients sustaining injuries and in $90 \%$ of patients with fractures were jumping on the trampoline with other children at the time of the accident. The most common fractures were supracondylar humeral fractures (29\%) and forearm fractures (25\%). Fractures of the proximal tibia occurred especially in younger children between 2-5 years of age.

Conclusions: Children younger than 5 years old are

Author Affiliations: Department of Pediatric Surgery (Klimek P, Juen D, Slongo T), Department of Diagnostic, Interventional and Pediatric Radiology, Inselspital, University of Bern, Switzerland (Stranzinger E, Wolf R)

Corresponding Author: Peter Michael Klimek, MD, Department of Pediatric Surgery University of Bern, Inselspital, CH- 3010 Bern, Switzerland (Tel: 00413163221 11; Fax: 00413163292 92; Email: peter.klimek@ksa.ch)

doi: 10.1007/s12519-013-0416-2

CChildren's Hospital, Zhejiang University School of Medicine, China and Springer-Verlag Berlin Heidelberg 2013. All rights reserved.

World J Pediatr, Vol 9 No 2 · May 15, 2013 · www.wjpch.com at risk for specific proximal tibia fractures ("Trampoline Fracture"). A child jumping simultaneously with other children has a higher risk of suffering from a fracture.

World J Pediatr 2013;9(2):169-174

Key words: fracture;

injuries;

prevention;

trampoline

\section{Introduction}

$\mathrm{B}$ ouncing on a trampoline is very popular among children, but several institutions have reported a significant increase in trampoline injuries in the last decades. ${ }^{[1-12]}$ Different expert panels have published the following safety recommendations, stressing that adult supervision is the most important safety measure to avoid trampoline accidents in younger children. Multiple jumpers can multiply the risk for injury. Children under age of 6 years should not be allowed on trampolines. The location of the trampoline is important. Safety nets are recommended to prevent injuries from falling. Springs and hooks should be covered with mats to avoid injury. ${ }^{[1,10-15]}$

Common trampoline injuries include sprains and strains of the ankles, fractures of the upper and lower extremities, accidents with spinal cord injuries as well as disabling head traumas, which can result in permanent paralysis. The most common causes of trampoline injuries according to the US Consumer Product Safety Commission are colliding with another person on the trampoline, landing improperly while jumping or doing stunts, falling off the trampoline and falling on the trampoline springs or frame. ${ }^{[3,6,7,9,16-27]}$

The aim of our study was to correlate data from a questionnaire given to the parents with the radiographic findings and to evaluate children at risk for trampolinerelated fractures. This study was conducted in collaboration with the Swiss Council for Accident Prevention and was approved by the local ethics committee. 


\section{Methods}

Between 2003 and 2009, all children (age $<16$ years) with backyard trampoline injuries were included in the study. All patients were presented at the Interdisciplinary Pediatric Emergency Unit, Departments of Pediatrics and Pediatric Surgery, Inselspital, University Hospital Bern, Switzerland. All patient records (type of injury, localization and treatment) and all radiographs were reviewed. Demographic data such as age, gender and month of the accident were included.

Each parent received by mail a questionnaire intended to evaluate the circumstances of the accident (the number of children, collision with other child, collision with frame or ground), the location of the trampoline and the type of ground under the trampoline (grass, gravel, stones/asphalt), the type of the trampoline (diameter, security net), parental supervision at the time of injury, playing time in minutes on the trampoline prior to the accident and the experience (in days) of the child using the trampoline.

The collected data from the questionnaire were correlated with the radiological findings (fracture type using the AO-classification, ${ }^{[28]}$ chronic repetitive stress changes, metaphyseal sclerosis, growth arrest lines and Salter Harris type fractures). All radiographs were blinded and retrospectively reviewed by a pediatric radiologist (with 8 years experience), who was unaware of the questionnaire's results. The study was

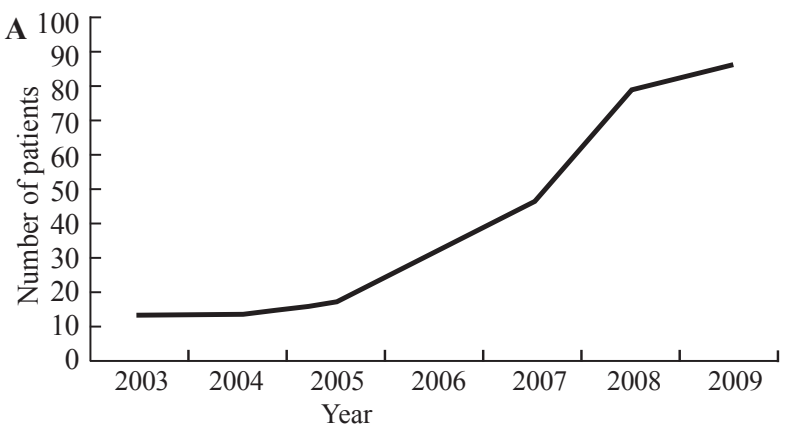

C

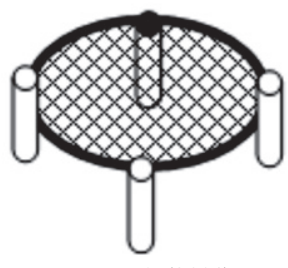

Type A $(29 \%)$

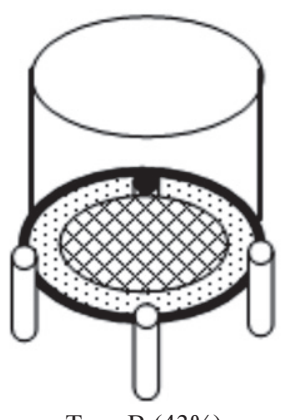

Type B (43\%) approved by the local ethics committee and conducted in collaboration with the Swiss Council for Accident Prevention.

\section{Results}

Between 2003 and 2009, we identified 286 children with trampoline-related injuries at our institution. The number of injuries increased from 13 patients in 2003 to 86 in 2009 (Fig. 1A). The median age was 7 years (range: $1-15$ years); 140 (49\%) patients were males, and $146(51 \%)$ females. The majority of the injuries and fractures (75\%) occurred between April and September (Fig. 1B). Of the 286 questionnaires sent, we received $171(60 \%)$ satisfactorily completed.

Of the 171 patients, 104 returned completed questionnaires (67 questionnaires were incomplete or the accident did not happen on a backyard trampoline). Most accidents happened at home (62 of 104, 60\%) followed by accidents at neighbors (35\%) and friends $(5 \%)$. Of these, $64 \%$ of the patients came directly to the emergency department of our clinic.

The most frequent causes of injury were accidents on the jumping plane (29 of 104, 28\%) followed by falling from the trampoline to the ground $(26 \%)$. Other causes included collision with another child $(20 \%)$ or with the frame (13\%), and $13 \%$ with unknown causes.

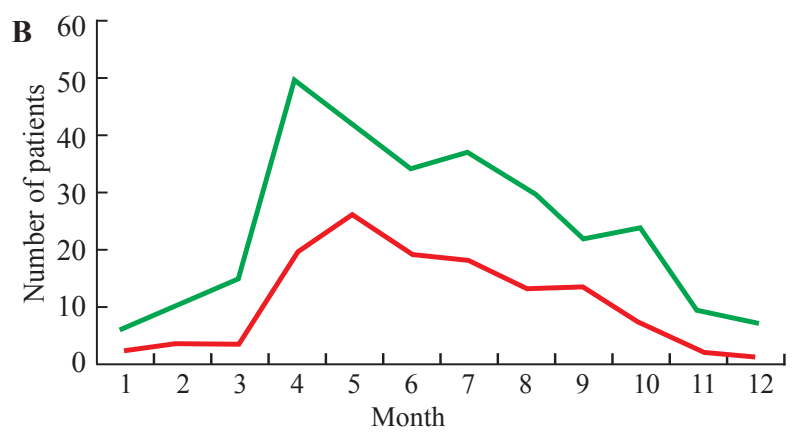

Fig. 1. A: increasing rate of trampoline-related injuries in our institution $(n=286)$; B: seasonal distribution of the injuries in our institution (green line, $n=286$ ) and fractures (red line, $n=126)$; $\mathbf{C}$ : types of trampoline and frequency of fractures $(n=51)$. 
In $85 \%$ of all cases reviewed the surface under and around the trampoline was grass, in $11 \%$ it was stone, and in $4 \%$ of the cases, gravel.

Over $75 \%$ of the children involved in an accident, and $90 \%$ of the children with fracture or other severe injury were jumping together with other children at the time of the accident. Parental supervision was evident in more than a quarter (28 of $104,27 \%$ ) of the cases. In $44 \%$ of the children, a trampoline with a security net was used outside of the frame (Type B, Fig. 1C). Only $36 \%$ of the injured children used a trampoline without net.

The majority of the accidents $(n=104)$ occurred on trampoline type A and B (35\%, 44\% respectively). Fractures $(n=51)$ were found in $29 \%$ of the cases in type $\mathrm{A}$ and in $43 \%$ in type $\mathrm{B} ; 12 \%$ of the accidents were counted on type $\mathrm{C}$ and $9 \%$ on type D. Fractures occurred in $20 \%$ on type $\mathrm{C}$ and in $8 \%$ on type D.

Twenty-eight of the $104(27 \%)$ accidents resulted from trampolines with a diameter less than $300 \mathrm{~cm}$. In 14 of the $28(50 \%)$ cases, we found one bone fracture. Seventy-two (69\%) accidents happened on trampolines with a diameter over $300 \mathrm{~cm}$. Of these, we found a bone fracture in 35 of $72(49 \%)$. The trampoline size was unknown in $4 \%$. Most of the accidents (47 of 104, $45 \%$ ) happened after 10 to 20 minutes of jumping, $16 \%$ within the first 10 minutes, $33 \%$ after 20 minutes, and $6 \%$ were unknown.

Forty-three of the $104(41 \%)$ injured children had a jumping experience less than 25 days. Among them, $23(53 \%)$ had a fracture. Twenty-eight $(27 \%)$ had a jumping experience of 25-100 days, and 16 (57\%) had a fracture. Only children with a jumping experience of over 100 days ( 26 children with 10 fractures) showed a decreased incidence of fractures $(38 \%)$.

We contacted all trampoline distributors in Switzerland to ask for the number of purchased trampolines in the study period. Only one of them answered that they had an increase of $466 \%$ in sales from 2003 to 2009.

\section{Radiographic results}

A fracture was found in 51 of the 104 patients. The most common fractures were upper extremity fractures $(n=35)$ such as supracondylar humeral fractures (15 of 35) and forearm fractures (13 of 35) (Fig. 2). Upper extremity fractures occurred frequently with direct impact on the ground (15 of 35), followed by collision with other children ( 9 of 35) and collision with frame (4 of 35). No chronic or repetitive changes of the upper extremities were found.

Lower extremity fractures were noted in 15 patients. Characteristic trampoline fractures of the lower extremities occurred in children aged 2-5 years in the proximal tibia metaphysis $(n=5)$ (Fig. 3 ). Special attention has to be paid on proximal tibia fractures in younger children (2-5 years old). Because of axial forces, the proximal tibia is at risk for impression fractures with anterior angulation and early closure of the growth plates. This fracture type may appear very subtle on initial radiographs and may be overlooked. In case of not conclusive radiographs and persistent pain of the tibia, follow-up radiographs after 7-10 days are recommended. In most of the children, a compression type fracture with anterior angulation of the growth plate was noted. There were always more than two children jumping on the trampoline when proximal tibia fractures occurred. Most of the proximal tibial fractures occurred in springtime between April and June.

Chronic repetitive changes [sclerosis of the metaphysis, growth arrest lines (Harris lines), widening
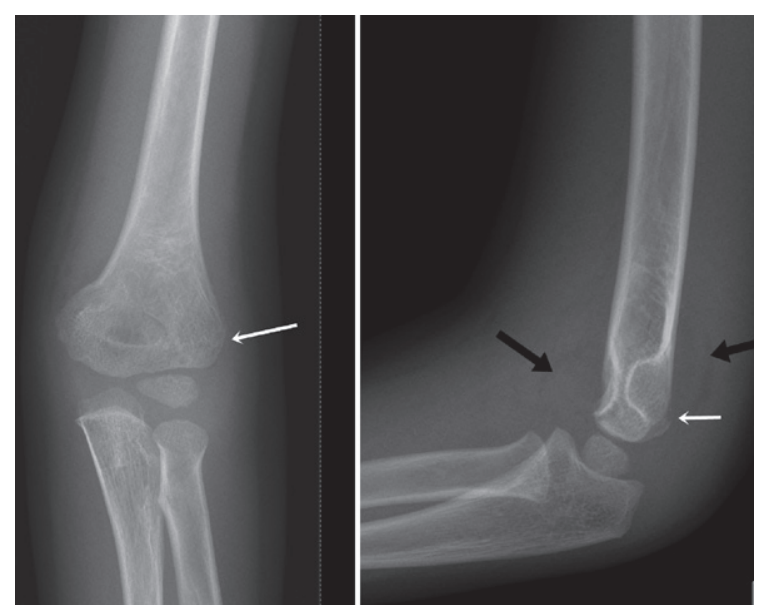

Fig. 2. A 6-year-old boy, dropped on the ground, with a supracondylar humeral fracture. Supracondylar fracture line (white arrow) and joint effusion (black arrow) is noted.

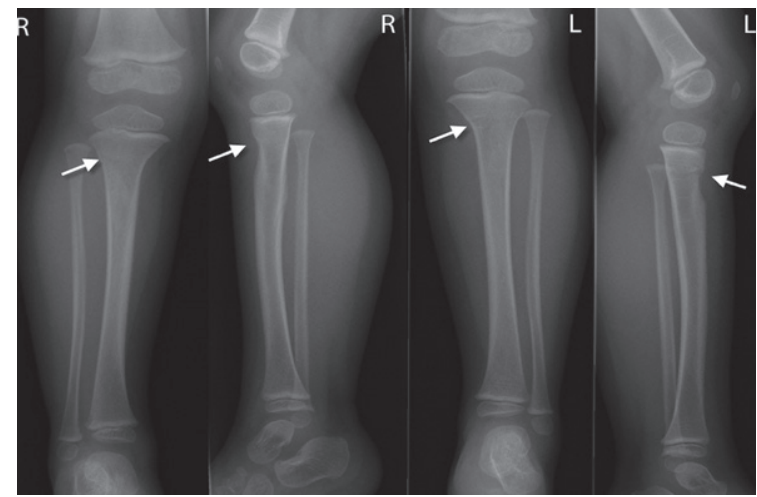

Fig. 3. A 2-year-old girl with characteristic right proximal tibia fracture (arrow). Three months later, the same fracture occurred on the left side. Mild anterior angulation of the growth plates on lateral radiographs is noted. 


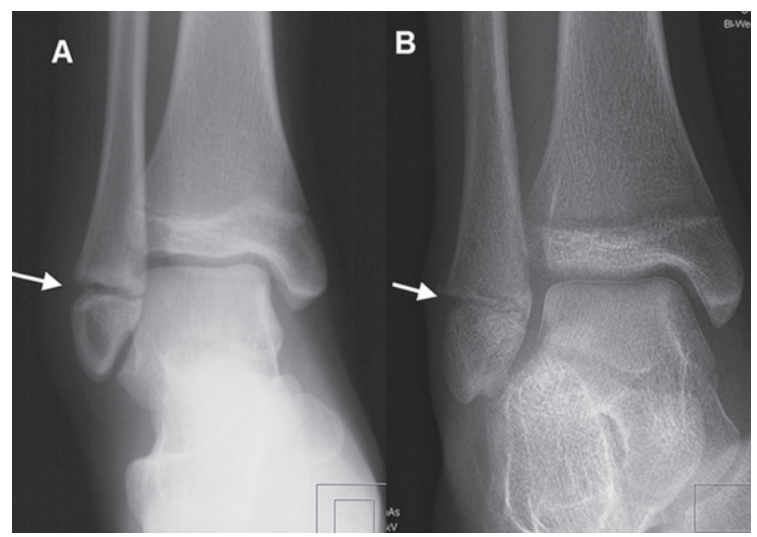

Fig. 4. A 13-year-old girl with supination trauma of the ankle. A: initial radiograph with widening of the fibular growth plate likely avulsion force (arrow) and swelling of the lateral malleolus; B: onemonth follow-up radiograph with narrowing of the growth plate of the fibula (arrow) and tibia (consistent with early closure).

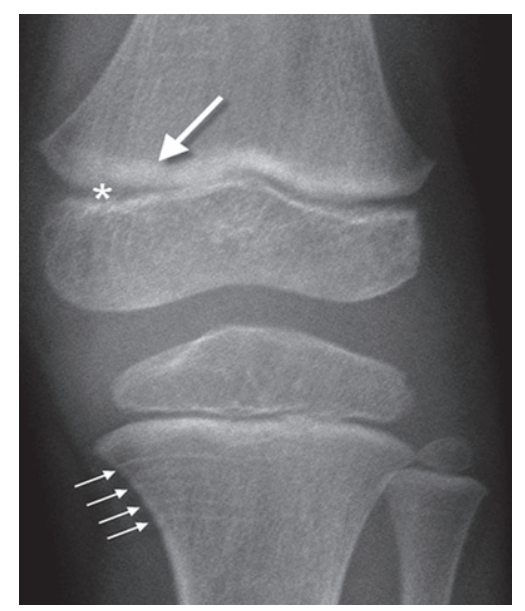

Fig. 6. A 4-year-old girl with sclerosis of the metaphysis (large white arrow), widening of the medial femoral growth plate (asterisk) and growth arrest lines (small white arrow).

of the growth plates] or Salter-Harris type fractures were found in 5 out of 15 children with fracture of the lower extremity. In two children, early closure of the growth plates was noted in follow-up radiographs (Figs. 4-6).

\section{Discussion}

The increasing popularity of backyard trampolines is associated with a rapidly rising incidence of injuries. Because of the increased rate of injuries, some authors regard the use of trampolines for recreational purposes as inherently unsafe and others even recommend banning backyard trampolines. ${ }^{[6,18,19,29,30]}$

Several authors suggest promoting parental and public awareness regarding the potential dangers of

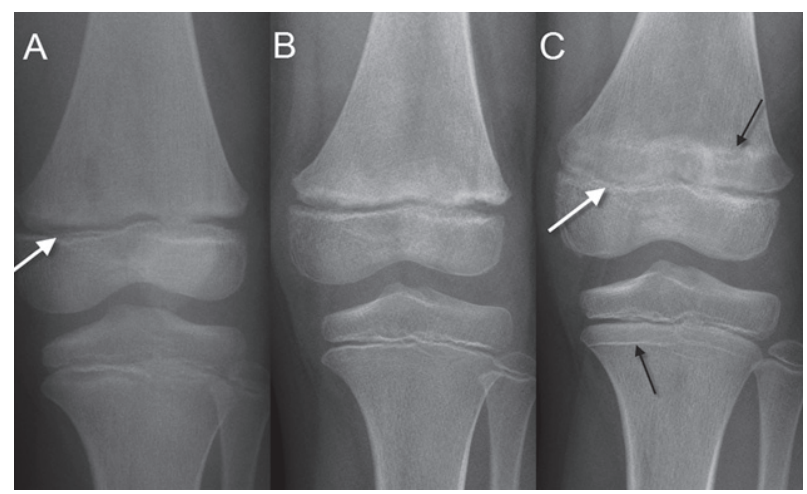

Fig. 5. A 7-year-old girl with Salter Harris Type I fracture of the distal femur (arrow); initial radiograph (A), follow-up radiographs after one month (B) and 7 months (C) with early closure of the medial growth plate (white arrow) of the femur and growth arrest lines (black arrow).

trampoline use.$^{[3,12,14]}$ Parental supervision is one of the most important factors in reducing risk of injuries on a trampoline. ${ }^{[31]}$ In our study, parental supervision was evident only in $27 \%$ of the accidents.

Another important risk factor is the number of children jumping simultaneously on the trampoline.$^{[6,10,11,19,20,32]}$ In our study, $75 \%$ of the accidents and more than $90 \%$ of the fractures occurred while more than one child was on the trampoline. Our data showed that neither a net nor larger diameter of the trampoline can protect children from severe injuries.

Shields et al ${ }^{[33]}$ also reported a similar level of severity of injuries for small and larger trampolines. Most of the bone fractures occurred on net-secured trampolines, thus a net may provoke children to more exaggerated jumping. A jumping experience over 100 days seems to decrease the risk for accidents and fractures, although this may not be statistically significant. Children between 2-6 years of age are at the highest risk to suffer a bone fracture due to trampoline accidents and most fractures occur in spring. Those facts may be due to age-related physiologic weakness and osteopenia in spring and early summertime caused by vitamin D deficiency and less activity during winter months, together with an increased activity in spring and early summer. ${ }^{[34,35]}$

\section{Radiographic findings}

Two different mechanisms may explain the different upper and lower extremity findings on radiographs. While the upper extremities show more fractures (supracondylar humerus fractures, forearm fractures, etc) due to direct impact on the ground or the frame of the trampoline, the lower extremities have a higher incidence of chronic repetitive changes. Axial forces to the ankle and knee joints may explain growth arrest lines, widening of the growth plates or sclerosis of the 
metaphysis. Although early closure after Salter type I fractures may occur, it is a very rare complication.

Multiple children on the trampoline may increase the axial forces to the knee joint and may cause the typical proximal tibia fractures in children less than 6 years of age ("Trampoline Fracture"). ${ }^{[17,36,37]}$ Swischuk and Boyer et al ${ }^{[17,37]}$ concluded that the most common lower extremity injury is a hyperextension injury of the knee, resulting in a compression fracture of the upper tibia. Those children are often between 2-6 years of age.

\section{Prevention}

In our opinion, a ban on the increasing and popular backyard trampolines is impossible. Sporting activity for children should be encouraged, but trampoline use should be undertaken with adult supervision and in accordance with safety procedures. Schoenholzer et $\mathrm{al}^{[38]}$ noted in 1962 the difficulty of the "benefit" of a sport. Considering factors like the development of physical strength, coordination or psychic well-being, trampoline jumping is an excellent opportunity to improve the perception of body and space.

Based on our data, the Swiss Council for Accident Prevention is preparing new safety recommendations for all parents for trampoline use ("bfu-Children's Mail") in Switzerland. The brochure is adapted to the particular development of children and informs parents about typical accident risks. "bfu-Children's Mail" includes 16 issues, starting with the birth of a child and to be delivered for free to the parents every 6 months.

In conclusion, the frequency of trampoline related injuries in children is increasing. Infants and children between 2-5 years of age are at higher risk to suffer from characteristic proximal metaphyseal tibia fractures. These children almost jump together with other children or persons on the trampoline at the time of injury, which may lead to higher axial forces to the lower extremities. Supervision by the parents is especially important in this age group. Chronic repetitive changes of growth plates in the lower extremities and early fusion of the growth plates may occur.

\section{Acknowledgements}

Andrea Kehl \& Marianne Kraus, study nurses. Othmar Brügger (Swiss Council for Accident Prevention) for the cooperation. Miriam Klimek for correction and translation.

\section{Funding: None.}

Ethical approval: The study was approved by the institutional ethics committee of the University Hospital of Bern.
Competing interest: None of the authors has financial or personal relationships with other persons or organizations that could inappropriately influence his or her actions.

Contributors: Klimek P and Juen D wrote the main body of the article. Stranzinger E and Wolf R provided advice on radiological aspects. Slongo $\mathrm{T}$ is the guarantor.

\section{References}

1 American Academy of Pediatrics. Committee on Injury and Poison Prevention and Committee on Sports Medicine and Fitness. Trampolines at home, school, and recreational centers. Pediatrics 1999;103:1053-1056.

2 Trampoline use in homes and playgrounds. Paediatr Child Health 2007; 12:501-511.

3 Bhangal KK, Neen D, Dodds R. Incidence of trampoline related pediatric large district general hospital in the United Kingdom: lessons to be learnt. Inj Prev 2006;12:133-134.

4 Chalmers DJ, Hume PA, Wilson BD. Trampolines in New Zealand: a decade of injuries. Br J Sports Med 1994;28:234-238.

5 Esposito PW, Esposito LM. The reemergence of the trampoline as a recreational activity and competitive sport. Curr Sports Med Rep 2009;8:273-277.

6 Furnival RA, Street KA, Schunk JE. Too many pediatric trampoline injuries. Pediatrics 1999;103:e57.

7 Hartmann KE, Calikoglu MG. Trampoline-related injuries to children. Pediatrics 1999;103:1311-1312; author reply 13121313.

8 Larson BJ, Davis JW. Trampoline-related injuries. J Bone Joint Surg Am 1995;77:1174-1178.

9 Loken-Dahle L. Trampoline-related injuries in children. Pediatrics 1999;103:1312; author reply 1312-1313.

10 Smith GA. Injuries to children in the United States related to trampolines, 1990-1995: a national epidemic. Pediatrics 1998;101:406-412.

11 Woodward GA, Furnival R, Schunk JE. Trampolines revisited: a review of 114 pediatric recreational trampoline injuries. Pediatrics 1992;89:849-854.

12 Wootton M, Harris D. Trampolining injuries presenting to a children's emergency department. Emerg Med J 2009;26:728731.

13 Rapp GF. II. Safety suggestions for trampoline use. Pediatr Ann 1978;7:730-731.

14 Rattya J, Serlo W. Using a safety net and following the safety instructions could prevent half the paediatric trampoline injuries. Eur J Pediatr Surg 2008;18:261-265.

15 Torg JS, Das M. Trampoline-related quadriplegia: review of the literature and reflections on the American Academy of Pediatrics' position statement. Pediatrics 1984;74:804-812.

16 Black GB, Amadeo R. Orthopedic injuries associated with backyard trampoline use in children. Can J Surg 2003;46:199201.

17 Boyer RS, Jaffe RB, Nixon GW, Condon VR. Trampoline fracture of the proximal tibia in children. AJR Am J Roentgenol 1986;146:83-85.

18 Brown PG, Lee M. Trampoline injuries of the cervical spine. Pediatr Neurosurg 2000;32:170-175.

19 Eberl R, Schalamon J, Singer G, Huber SS, Spitzer P, Hölwarth ME. Trampoline-related injuries in childhood. Eur J Pediatr 2009;168:1171-1174.

20 Esposito PW. Trampoline injuries. Clin Orthop Relat Res 


\section{3;(409):43-52.}

21 Hammer A, Schwartzbach AL, Paulev PE. Trampoline training injuries - one hundred and ninety-five cases. Br J Sports Med 1981;15:151-158.

22 Hume PA, Chalmers DJ, Wilson BD. Trampoline injury in New Zealand: emergency care. Br J Sports Med 1996;30:327-330.

23 Kravitz H. Problems with the trampoline: I. Too many cases of permanent paralysis. Pediatr Ann 1978;7:728-729.

24 McDermott C, Quinlan JF, Kelly IP. Trampoline injuries in children. J Bone Joint Surg Br 2006;88:796-798.

25 Nysted M, Drogset JO. Trampoline injuries. Br J Sports Med 2006;40:984-987.

26 Rapp GF, Nicely PG. Trampoline injuries. Am J Sports Med 1978;6:260-271.

27 Shankar A, Williams K, Ryan M. Trampoline-related injury in children. Pediatr Emerg Care 2006;22:644-646.

28 Matter P, Buhler M. 35 years fracture documentation by the Working Group for Osteosynthesis Problems (AO). Swiss Surg 1995;57-60.

29 Hammer A, Schwartzbach AL, Paulev PE. Some risk factors in trampolining illustrated by six serious injuries. Br J Sports Med 1982;16:27-32.

30 Leonard H, Joffe AR. Children presenting to a Canadian hospital with trampoline-related cervical spine injuries. Paediatr Child
Health 2009; $14: 84-88$

31 Smith GA, Shields BJ. Trampoline-related injuries to children. Arch Pediatr Adolesc Med 1998;152:694-699.

32 Bogacz A, Paterson B, Babber A, Menelaws S, Drew T. Trampoline injuries. How to avoid injury. BMJ 2009;338: b2197.

33 Shields BJ, Fernandez SA, Smith GA. Comparison of minitrampoline- and full-sized trampoline-related injuries in the United States, 1990-2002. Pediatrics 2005;116:96-103.

34 Bloom E, Klein EJ, Shushan D, Feldman KW. Variable presentations of rickets in children in the emergency department. Pediatr Emerg Care 2004;20:126-130.

35 Chapman T, Sugar N, Done S, Marasigan J, Wambold N, Feldman K. Fractures in infants and toddlers with rickets. Pediatr Radiol 2010;40:1184-1189.

36 Clare PE. Trampoline injuries to the lower extremity. Two case reports. Am J Sports Med 1978;6:141-142.

37 Swischuk LE. Jumped off the trampoline: fell on knee: pain. Pediatr Emerg Care 2009;25:366-367.

38 Schoenholzer G. Trampoline jumping from a medical viewpoint. Schweiz Z Sportmed 1962;10:6-22.

Received February 29, 2012 Accepted after revision June 20, 2012 